\title{
Angiotensin receptor-neprilysin inhibition in patients with acute decompensated heart failure: an expert consensus position paper
}

\author{
Argyrios Ntalianis $^{1}$ (1) . Christina Chrysohoou ${ }^{2}$. George Giannakoulas ${ }^{3}$. Grigorios Giamouzis ${ }^{4}$. \\ Apostolos Karavidas $^{5} \cdot$ Aikaterini Naka $^{6}$. Constantinos H. Papadopoulos ${ }^{7}$. Sotirios Patsilinakos ${ }^{8}$. John Parissis ${ }^{9}$. \\ Dimitrios Tziakas ${ }^{10}$. John Kanakakis ${ }^{11,12}$
}

Accepted: 20 April 2021 / Published online: 1 May 2021

(c) The Author(s), under exclusive licence to Springer Science+Business Media, LLC, part of Springer Nature 2021

\begin{abstract}
The short-term mortality and rehospitalization rates after admission for acute heart failure (AHF) remain high, despite the high level of adherence to contemporary practice guidelines. Observational data from non-randomized studies in AHF strongly support the in-hospital administration of oral evidence-based modifying chronic heart failure (HF) medications (i.e., b-blockers, ACE inhibitors, mineralocorticoid receptor antagonists) to reduce morbidity and mortality. Interestingly, a well-designed prospective randomized multicenter study (PIONEER-HF) showed an improved clinical outcome and stress/ injury biomarker profile after in-hospital administration of sacubitril/valsartan (sac/val) as compared to enalapril, in hemodynamically stable patients with AHF. However, sac/val implementation during hospitalization remains suboptimal due to the lack of an integrated individualized plan or well-defined appropriateness criteria for transition to oral therapies, an absence of specific guidelines regarding dose selection and the up-titration process, and uncertainty regarding patient eligibility. In the present expert consensus position paper, clinical practical recommendations are proposed, together with an action plan algorithm, to encourage and facilitate sac/val administration during hospitalization after an AHF episode with the aim of improving efficiencies of care and resource utilization.
\end{abstract}

Keywords Acute heart failure $\cdot$ Sacubitril/valsartan $\cdot$ Hospitalization

\section{Introduction}

Heart failure (HF) is a complex clinical syndrome characterized by abnormalities in cardiac structure and function, dynamic remodeling, and perturbations of the

John Parissis received honoraria for lectures from Servier, Pfizer, Novartis, Orion Pharma, and Astra Zeneca.

Argyrios Ntalianis

arg_nt@yahoo.gr

1 Heart Failure \& Cardio-Oncology Unit, Alexandra Hospital, Athens, Greece

2 1st Cardiology Clinic, University of Athens, Hippokratio Hospital, Athens, Greece

3 1st Cardiology Clinic, Aristotle University of Thessaloniki, AHEPA General Hospital, Thessaloniki, Greece

4 University General Hospital of Larissa, University of Thessaly, Larissa, Greece

5 Cardiology Clinic, Gennimatas General Hospital, Athens, Greece neurohormonal axis [1]; it remains one of the leading causes of hospitalization. Nearly $44 \%$ of HF patients are readmitted for cardiac and non-cardiac related conditions within 1 year of discharge and the duration of readmission ranges between 4 and 6 days. It is worth noting that almost half of rehospitalizations are expected to occur in the first 12 weeks post-discharge during the so-called 'vulnerable' phase [2].

6 University Cardiology Clinic, University of Ioannina, Ioannina, Greece

7 1st Cardiology Clinic, Red Cross Hospital, Athens, Greece

8 Cardiology Clinic, Konstantopoulio General Hospital, Athens, Greece

9 Heart Failure Unit, Attikon Hospital, Athens, Greece

10 University Cardiology Clinic, Democritus University of Thrace, Alexandroupoli, Greece

11 Department of Clinical Therapeutics, Catheterization Laboratory, University of Athens, Athens, Greece

12 Hellenic Society of Cardiology, Athens, Greece 
Retrospective cohorts and registries show a decline in chronic HF mortality rates from 100 deaths per 100,000 population in 1987 to 50 deaths per 100,000 population in 2008, but with a parallel increase in HF-related hospitalizations [3]. Hospitalized patients with a diagnosis of acute HF (AHF) have a higher risk of mortality as compared with stable patients with chronic HF, probably because of an overactivation of the neurohormonal axis; this further emphasizes the need for early institution and optimization of neurohormonal inhibition therapies in these patients $[4,5]$. In fact, the continuation of the HF-modifying medications even in lower doses during hospitalization in chronic HF patients and the early in-hospital initiation of these agents for de novo HF patients are associated with symptom improvement and a lower rate of short- and long-term cardiovascular events. [6-9].

\section{Unmet needs in AHF therapy}

\section{(i) Therapeutic options are limited}

The generally accepted therapeutic options for AHF recommended by both the European [10] and American Society of Cardiology [11] are limited to three targets:

(a) afterload reduction with intravenous vasodilators;

(b) preload reduction and decongestion with intravenous loop diuretics; and

(c) contractility modulation with inotropes for hypoperfusion/hypotension.

Interestingly, these recommendations mostly come from consensus of experts and/or small, retrospective studies and registries; thus, their level of evidence is very weak. Very recently, the GALACTIC Study—a randomized, open label, multicenter study - did not show any clinical benefits in all-cause mortality and AHF rehospitalization at 180 days with an early intensive and sustained vasodilatation strategy compared with usual care [12]. A number of other doubleblinded prospective randomized studies failed to reveal any significant efficacy for several novel intravenous and oral agents targeting different pathophysiological pathways in patients with AHF [13], with the exception of the relatively recent PIONEER-HF study which showed positive results for sacubitril/valsartan (sac/val) [14]. Perhaps, the heterogeneity of presenting phenotypes and complex pathophysiology of AHF could explain to some extent the disappointing results of those studies. In contrast, evidence from openlabel randomized and observational studies and registries support the use of the 'classic' HF-modifying treatment with b-blockers, angiotensin-converting enzyme inhibitors (ACE-I), angiotensin II receptor blockers (ARBs), and mineralocorticoid receptor antagonists (MRAs) during hospitalization for AHF [15].

Based on these findings, current guidelines strongly recommend (class I C) HF-modifying therapy in patients with reduced ejection fraction (EF) hospitalized with a diagnosis of AHF as soon as hemodynamic stability is achieved, with the goal of attaining a stable clinical course [11]. Accordingly, hospitalization for AHF provides an opportunity to introduce or optimize guideline-directed medical treatment $[16,17]$.

\section{(ii) Transition from intravenous to oral medications is challenging}

A daily clinical trajectory check is required to identify the ideal timing for the transition from intravenous to oral medications. This process is extremely challenging since much ambiguity exists in defining strict criteria for clinical stability. Drug priority, dosage, and up-titration may also represent additional barriers during the transition phase.

Clinical stability is synonymous with hemodynamic stability, which entails effective decongestion and euvolemia [18]. Clinical and laboratory criteria exist to characterize the fluid volume status and the response to intravenous diuretics. Although it is possible to distinguish between hypervolemia and euvolemia in most cases, the distinction between euvolemia and hypovolemia sometimes remains speculative. Undoubtedly, it is extremely clinically important to identify hypovolemia since hypovolemic patients may respond with severe hypotension after the administration of ACE-I or ARBs [15].

\section{Sac/val in chronic HF}

The PARADIGM-HF trial was the first prospective double-blinded multicenter randomized trial in symptomatic patients with chronic HF and reduced EF (HFrEF) $(\leq 40 \%)$ to demonstrate a $20 \%$ relative risk reduction in the composite endpoint of cardiovascular death or hospitalization for HF, a $20 \%$ reduction in sudden death, and a $16 \%$ reduction in allcause mortality with sac/val as compared to enalapril [19]. These clinically relevant beneficial effects are attributed to a significant improvement of the left ventricular diastolic function and arterio-ventricular coupling [20], a significant decrease of NT pro-BNP, and notably an antiapoptotic, antifibrotic [21, 22], and anti-remodeling effect [23]. Sac/val also exerts renoprotective actions [24] and unique metabolic effects as it improves glucose tolerance in patients with HF and diabetes [25]. Interestingly, data from PARADIGMHF showed not only better efficacy with sac/val but also an acceptable safety profile. Although hypotension was more common with sac/val as compared with enalapril, this did not lead to a higher incidence of permanent discontinuation 
[26]. The salient results of PARADIGM-HF [19] which were further substantiated by the findings of PROVE-HF [23] and EVALUATE-HF [20] have been recently highlighted in an American College of Cardiology expert consensus update on HF treatment optimization. It was concluded that sac/val is the 'preferred' renin-angiotensin antagonist in patients with HFrEF stage $\mathrm{C}$ and ACE-I/ARBs should only be considered in cases with contraindications, intolerance, or inaccessibility to sac/val. [27]

Lastly, the role of sac/val continues to be explored in patients with advanced HF (NYHA IV) in the context of a prospective, multicenter, double-blinded, double-dummy trial. Unfortunately, the LIFE trial was halted prematurely because of the COVID-19 pandemic and the data analysis plan has changed. [28]

\section{Sac/val in AHF}

\section{a. TRANSITION study}

The TRANSITION study [29], an international prospective multicenter randomized open-label clinical trial, enrolled 1002 adult patients with HFrEF ( $\mathrm{EF} \leq 40 \%)$, hospitalized with a primary diagnosis of AHF, New York Heart Association functional class II-IV, and systolic blood pressure $(\mathrm{SBP}) \geq 100 \mathrm{mmHg}$. Eligible candidates were randomized to oral sac/val either during hospitalization or early ( 2 weeks) post-discharge. Interestingly, in the majority of the cases ( 85\%), the lowest sac/val dose (24/26 mg twice daily) was selected by the enrolling physicians as the starting drug dose. The target dose pre-specified by protocol (103/97 mg twice daily) was achieved in $~ 50 \%$ of the patients. There was no significant difference between pre- and post-discharge sac/val administration in terms of the likelihood in achieving the target dose. A higher probability of sac/val up-titration to target dose was found among patients with normal SBP $(\geq 120 \mathrm{mmHg}$ ), preserved renal function (eGFR $>60 \mathrm{~mL} /$ $\mathrm{min} / 1.73 \mathrm{~m}$ ), and de novo HF [29, 30]. In a post hoc efficacy analysis, pre-discharge initiation of sac/val as compared to post-discharge was associated with a higher reduction of NTproBNP levels and higher NT-ProBNP favorable reduction response [31].

Despite the inherent limitations of the study [32], TRANSITION showed emphatically that soon after hospitalization for AHF, it is feasible to achieve effective doses of sac/val in clinically stable patients within a short timeframe.

\section{b. PIONEER-HF study}

PIONEER-HF [14], a prospective double-blinded multicenter randomized study, showed very promising outcome results with sac/val in the setting of AHF. In short, 881 patients with HFrEF admitted for AHF were randomly assigned to either sac/val or enalapril during hospitalization. Along with clinical criteria, elevated BNP ( $\geq 400 \mathrm{pg} / \mathrm{dL})$ or NT-proBNP $(\geq 1600 \mathrm{pg} / \mathrm{dL})$ values before randomization had to be met in order for a patient to be considered eligible for the study (Table 1). The median duration of hospitalization was $\sim 5$ days. It is worth noting that more than $30 \%$ of the patients were diagnosed with de novo HFrEF and only $\sim 50 \%$ reported previous use of ACE-I or ARBs $[14,33]$. Serious adverse events were comparable between the study groups.

The main finding of the study was a significantly greater reduction of NT-proBNP values in the group of sac/val at 8 weeks post-discharge. Notably, these lower NT-proBNP levels were evident from the first week of treatment with sac/val [33].

Furthermore, in an exploratory analysis of the clinical outcomes, sac/val further reduced the risk of the composite of cardiovascular death and rehospitalization for HF (by $42 \%$ ); this risk reduction was principally driven by a $36 \%$ decrease of rehospitalizations for HF [34]. Notably, an early separation of the event curves was observed from the first week of the study in favor of sac/val. The benefit from sac/ val was evident across all dose levels tested [35]. Furthermore, the positive effect of sac/val on NT-proBNP and clinical outcomes was independent of a previous HF diagnosis or prior ACE-I/ARB treatment [36].

Several pathophysiological mechanisms have been implicated in this favorable prognosis, including the reduction of myocardial injury and hemodynamic stress reflected by a greater decline in high sensitivity troponin $\mathrm{T}$ and soluble ST2 [33].

Even late post-discharge switch from enalapril to sac/val had a significant impact on NT-proBNP reduction in the open-label extension of PIONEER-HF, but unfortunately, this effect did not translate into a significantly better outcome [37]. This implies that sac/val initiation early during hospitalization is a key factor in improving clinical outcome since most of the clinical benefit is gained in the first 2 months after discharge.

\section{In-hospital sac/val and cost-effectiveness}

From a healthcare system perspective, initiation of sac/val during hospitalization saves $\$ 452$ per year as compared with continuing enalapril and $\$ 811$ per year as compared with a strategy of late post-discharge initiation [38]. Moreover, initiation of sac/val as an inpatient was associated with an incremental cost-effectiveness ratio of $\$ 21,532$ per qualityadjusted life-year as compared with continued enalapril treatment over a lifetime [38]. 
Table 1 PIONEER HF's modified criteria for sac/val initiation

\begin{tabular}{|c|c|}
\hline PIONEER-HF criteria & Modified criteria \\
\hline $\begin{array}{l}\text { Currently hospitalized for a primary diagnosis of HF, including symp- } \\
\text { toms and signs of fluid overload }\end{array}$ & $\begin{array}{l}\text { Currently hospitalized for a primary diagnosis of HF, including symp- } \\
\text { toms and signs of fluid overload and/or hypoperfusion }\end{array}$ \\
\hline $\begin{array}{l}\text { Randomized no earlier than } 24 \mathrm{~h} \text { and up to } 10 \text { days after initial pres- } \\
\text { entation while still hospitalized, as long as they meet the following } \\
\text { definition of hemodynamically stable status }\end{array}$ & Consider sac/val initiation when the patient is clinically stable \\
\hline $\begin{array}{l}\text { Hemodynamically stable status defined by: } \\
\text { (a) maintenance of } \mathrm{SBP} \geq 100 \mathrm{~mm} \mathrm{Hg} \text { for the preceding } 6 \mathrm{~h} \text { in the } \\
\text { absence of symptomatic hypotension } \\
\text { (b) no increase (i.e., intensification) in the dose of IV diuretics or use } \\
\text { of IV vasodilators within the last } 6 \mathrm{~h} \text { and } \\
\text { (c) no use of IV inotropes for } 24 \mathrm{~h} \text { prior to randomization }\end{array}$ & $\begin{array}{l}\text { Clinical stability criteria (all } 4 \text { criteria below have to be applied): } \\
\text { (a) } \mathrm{SBP} \geq 100 \mathrm{mmHg} \text { for the last } 6-12 \mathrm{~h} \text { and for at least } 3 \mathrm{BP} \text { measure- } \\
\text { ments } \\
\text { (b) Euvolemic status } \\
\text { (c) Unchanged dose of intravenous (and preferably oral) diuretics for } \\
\text { the last } 6-12 \mathrm{~h} \\
\text { (d) Withdrawal of intravenous vasodilators, inotropes or vasopressors } \\
\text { for the last } 6-12 \mathrm{~h}^{\mathrm{a}}\end{array}$ \\
\hline $\begin{array}{l}\text { Left ventricular } \mathrm{EF} \leq 40 \% \text { within the past } 6 \text { months by echocardiogra- } \\
\text { phy, MUGA, CT scanning, MRI or ventricular angiography provided } \\
\text { no subsequent study documented an } \mathrm{EF}>40 \%\end{array}$ & $\begin{array}{l}\text { Left ventricular } \mathrm{EF} \leq 40 \% \text { assessed by echocardiography during the cur- } \\
\text { rent hospitalization for AHF }\end{array}$ \\
\hline $\begin{array}{l}\text { Elevated NT-proBNP } \geq 1600 \mathrm{pg} / \mathrm{mL} \text { or } \mathrm{BNP} \geq 400 \mathrm{pg} / \mathrm{mL} \text { during the } \\
\text { current hospitalization }\end{array}$ & Natriuretic peptides are optional before the initiation of sac/val \\
\hline $\begin{array}{l}\text { Starting dose of sac/val: } \\
\text { (a) } 49 / 51 \mathrm{mg} \text { BID for patients with SBP } \geq 120 \mathrm{mmHg} \\
\text { (b) } 24 / 26 \mathrm{mg} \text { BID for patients with SBP } 100-120 \mathrm{mmHg}\end{array}$ & $\begin{array}{l}\text { Starting dose of sac/val: } \\
\text { (b) } 24 / 26 \mathrm{mg} \text { BID for all patients with } \mathrm{SBP} \geq 100 \mathrm{mmHg}\end{array}$ \\
\hline
\end{tabular}

$A H F$ acute heart failure, $B I D$ twice a day, $B N P$ brain natriuretic peptide, $B P$ blood pressure, $E F$ ejection fraction, Sac/Val sacubitril/valsartan, $S B P$ systolic blood pressure

${ }^{\mathrm{a}}$ For milrinone longer cessation time is required $(\sim 24-30 \mathrm{~h})$, and for levosimendan, closer supervision is needed normally beyond 6-12 $\mathrm{h}$ because of its long-acting metabolites

\section{ESC and ACC expert reports for sacubitril-valsartan in AHF}

The findings of PIONEER-HF [14] and TRANSITION [29] have recently been implemented by a panel of experts from the Heart Failure Association of the European Society of Cardiology, concluding that initiation of sac/val rather than ACE-I or ARBs may be considered for patients hospitalized with new-onset HF or decompensated HF to reduce the short-term risk of adverse events and to simplify management by avoiding the need to titrate ACE-I first and then switch to sac/val [2]. Accordingly, an American College of Cardiology expert consensus clearly outlines the extensive experience with initiation of ACE-I and ARBs in hospitalized patients with AHF but also gives emphasis on PIONEER-HF results and the benefit gained from the initiation of sac/val during hospitalization [5]. Lastly, in a very recent American College of Cardiology consensus, it is stated that improvement in early clinical outcomes with in-hospital sac/val initiation would be lost in a scenario of ACE-I/ARBs pretreatment and thus a strategy of 'de novo' sac/val initiation is advocated [27].

\section{Scope of the current consensus document}

Sac/val is prescribed in a small minority of cases (1-2\%) of AHF despite its attractive safety and efficacy profile [39]. The reluctance surrounding routine sac/val implementation during hospitalization may be attributed to the lack of detailed practical recommendations and the complexity of dose selection and up-titration algorithms proposed by some previously published papers [40].

The present expert consensus position paper gives practical insights on sac/val implementation during hospitalization for AHF. Recommendations and criteria for sac/val initiation, dosage, up-titration process, recognition of side effects, and monitoring are proposed and thoroughly discussed.

\section{Consensus recommendations}

\section{All patients with HFrEF admitted with AHF should be considered candidates for sac/val (Fig. 1)}

From the first day of admission, every patient with HFrEF who is hospitalized with AHF should be routinely screened and 
considered as a potential candidate for sac/val. The Get With The Guidelines-HF registry came to a similar conclusion since the majority of AHF patients enrolled in the registry and normally encountered in routine practice had characteristics and clinical outcomes that were comparable with those of patients eligible for PIONEER-HF [41]. To facilitate screening and the selection process for sac/val candidates, it is imperative for all the physicians to set up AHF hospitalization protocols, databases, and guideline-directed HF therapies check-lists. Hence, better adherence to current guidelines regarding treatment recommendations is anticipated. $[2,11,16]$

\section{Criteria for in-hospital sac/val initiation}

The panel of experts discussed PIONEER-HF's inclusion and exclusion criteria in detail and modified them accordingly, to simplify procedures and ease decision making for in-hospital sac/val administration (Table 1).

\section{Low cardiac output syndrome}

According to PIONEER-HF inclusion criteria, patients hospitalized with AHF who showed symptoms and signs of fluid overload but not hypoperfusion were eligible for sac/val. Interestingly, only a small minority of the study patients (7.7\%) required inotropes during hospitalization, possibly because of hypoperfusion or hypotension. Need for inotropes during hospitalization reflects a high-risk patient profile with an unfavorable prognosis [42-45], which may, however, benefit from neurohormonal axis inhibition $[9,46]$. As mentioned above, current guidelines strongly support the concept of continuation or initiation of the oral evidence-based HF-modifying therapies after hemodynamic stabilization for all the patients with AHF without any exception [10]. Thus, it was unanimously decided by the experts to expand the recommendation for in-hospital sac/val to patients with hypoperfusion, on the condition that strict criteria for clinical stability are fulfilled.

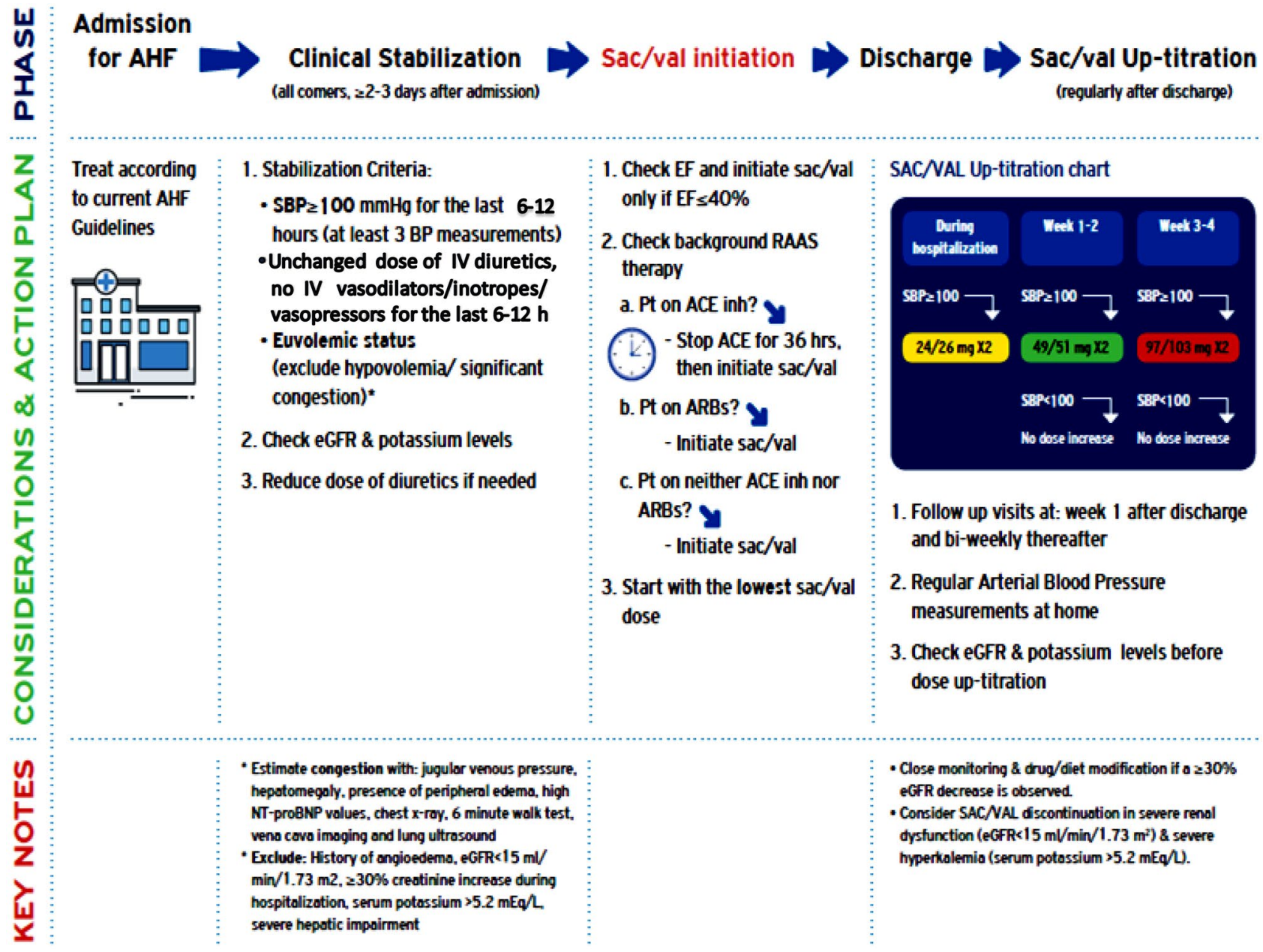

Fig. 1 Proposed algorithm for sac/val initiation during hospitalization for AHF and post discharge up-titration protocol. ACEinh: angiotensin-converting enzyme inhibitor, AHF: acute heart failure, ARB:

angiotensin II receptor blocker, eGFR: estimated glomerular filtration rate, SBP: systolic blood pressure, Sac/val: sacubitril/valsartan 


\section{Clinical stability criteria}

PIONEER-HF's clinical stability criteria have been reconsidered on the basis of safety, practicality, and applicability. The resolution of congestion requires intravenous agents (diuretics, vasodilators, and inotropes), which may result in a significant blood volume redistribution as well as cardiac output and blood pressure variations. For this reason, ample time is required for hemodynamic stabilization. The optimal timing for the transition to oral medications is variable, differing from patient to patient, and is mostly determined by the clinical phenotype and patient profile at admission (congestion, hypoperfusion, hypotension), presence of comorbidities (i.e., renal dysfunction, anemia), and response to treatment [5]. Empirically, clinical stability is achieved within 2-3 days after admission for the less severe cases with AHF. In PIONEER-HF, the median duration of index hospitalization was 5.2 days (interquartile range, 4.09 to 7.24 ), which means that clinical stability and transition to oral therapies were accomplished earlier during hospitalization. Taking into account the low rate of admission to intensive care unit (11\% of cases) and the small number of patients who required inotropic support, it can be assumed that the population enrolled in PIONEER-HF represents moderate forms of AHF. However, in everyday clinical practice, several and even extreme AHF phenotypes are encountered; individualization and appropriate tailoring of the treatment options are therefore required.

According to the panelists, all the following criteria need to be met to consider a patient with AHF eligible for sac/val:

(i) Systolic blood pressure higher or equal to $100 \mathrm{mmHg}$ in at least 3 measurements during the last $6-12 \mathrm{~h}$, provided that no signs of low-cardiac output syndrome and peripheral hypoperfusion exist;

(ii) Euvolemic status;

(iii) Unchanged dose of intravenous (and preferably oral) diuretics for the last $6-12 \mathrm{~h}$; and

(iv) Withdrawal of intravenous vasodilators, inotropes, or vasopressors during the last 6-12 h.

\section{Sac/val and volume status}

Clearly, the most critical and challenging step before considering sac/val administration is to ensure that the patient is euvolemic. Patients with hypervolemia and residual congestion can easily deteriorate and thus sac/val is considered a relative contraindication. Theoretically, sac/val could be reserved with caution for selected cases with hypervolemia, refractory edema, and normal or high blood pressure, with the aim of relieving congestion via natriuresis and improving cardiac output via afterload reduction. Clinical, biochemical, radiographic, and echocardiographic parameters, as well as exercise capacity, are surrogates for the estimation of fluid volume status and for the identification of residual congestion. Hypervolemia and residual congestion are characterized by hepatomegaly, presence of peripheral edema, distention of jugular veins, high values of BNP $(>100 \mathrm{pg} /$ $\mathrm{dL})$ or NT-proBNP ( $>400 \mathrm{pg} / \mathrm{dL})$, inspiratory diameter of inferior vena cava $>12 \mathrm{~mm}$ and/or lateral $E / e^{\prime}>12$ in heart ultrasound, and diffuse B-lines in chest $\mathrm{x}$-ray and lung ultrasound [47]. Unquestionably, ventricular filling pressures are considered the most accurate indices to diagnose fluid overload. It should be noted that the diagnostic accuracy of heart ultrasound to assess right and left ventricular filling pressures is acceptable [48] but not optimal, especially for cases with mild/moderate congestion due to intraobserver variability and other technical issues. [47]

Although hypervolemia is considered a relative contraindication, hypovolemia is an absolute contraindication for inhospital sac/val administration because of a higher risk for hypotension. Distinguishing euvolemia from hypovolemia is sometimes challenging. Borderline blood pressure, reduced skin turgor, orthostatism, hypernatremia, significant increase of hematocrit and total protein, oliguria, and a creatinine increase of more than $30 \%$ from the baseline creatinine values at admission are proposed as surrogate markers of a clinically significant reduction of the effective blood volume [49]. Absolute and relative contraindications for in-hospital sac/val administration are shown in Table 2. Remarkably, moderate renal and hepatic dysfunction and hyperkalemia should prompt close monitoring of patients considered for sac/val.

\section{Transition from intravenous agents to sac/val}

For most of the intravenous agents commonly used in AHF (vasodilators, diuretics, inotropes, vasopressors) with the exception of milrinone and torsemide, the pharmacological half-life is short (from $2 \mathrm{~min}$ for dobutamine to $1.3 \mathrm{~h}$ for levosimendan) [50, 51]. It is estimated that $97 \%$ of a drug is eliminated when five half-lives have passed, in the absence of severe renal or hepatic dysfunction. Consequently, only negligible concentrations of these agents are expected after $6-12 \mathrm{~h}$ of cessation. Practically, the elimination time for $97 \%$ of dobutamine is only $10 \mathrm{~min}$, for levosimendan, it is $6.5 \mathrm{~h}$ [50], and for milrinone and torsemide, it can range from 10 to $30 \mathrm{~h}[51,52]$. For these reasons, we recommend a minimum of 6-12 h withdrawal of vasodilators, inotropes, or vasopressors before sac/val initiation. Undoubtedly, longer cessation time $(\sim 24-30 \mathrm{~h})$ is required for milrinone. From our experience, closer supervision is sometimes needed for patients treated with levosimendan, beyond the first $6-12 \mathrm{~h}$ of discontinuation, because of its long-acting circulating metabolites with expected half-life of $80 \mathrm{~h} \mathrm{[50].}$ 
Table 2 Contraindications and special warnings and precautions for sac/val use

Sac/val contraindications (use is not indicated)
1. Hypersensitivity to the active substances or to any of the excipients
2. Concomitant use with ACE-I. Sac/val must not be administered until
36 h after discontinuing ACE-I therapy
3. Known history of angioedema related to previous ACE-I or ARB
therapy
4. Hereditary or idiopathic angioedema
5. Concomitant use with aliskiren-containing medicinal products in
patients with diabetes mellitus or in patients with renal impairment
(eGFR $<60 \mathrm{~mL} / \mathrm{mi} / 1.73 \mathrm{~m}^{2}$ )
6. Severe hepatic impairment, biliary cirrhosis and cholestasis (Child-
Pugh C classification)
7. Second and third trimester of pregnancy
8. Serum potassium $>5.5 \mathrm{mmol} / \mathrm{L}$
9. Significant hypovolemia

Sac/val special warnings and precautions for use (use with caution)

1. eGFR $<30 \mathrm{~mL} / \mathrm{min} / 1.73 \mathrm{~m}^{2}$. There is no experience in patients with end-stage renal disease and use of sac/val is not recommended

2. Incidence of worsening renal function, defined as an increase in serum creatinine of $\geq 0.5 \mathrm{mg} / \mathrm{dL}$ and a decrease of eGFR by at least $30 \%$

3. Moderate hepatic impairment (Child-Pugh B classification, or AST/

ALT levels more than twice above the upper normal range)

4. Hypervolemia

$A C E-I$ angiotensin-converting enzyme inhibitor, $A L T$ alanine transferase, $A R B$ angiotensin II receptor Blocker, $A S T$ aspartate transferase, $e G F R$ estimated glomerular filtration rate, Sac/val sacubitril/valsartan

\section{Left ventricular ejection fraction}

Only patients with an $\mathrm{EF} \leq 40 \%$ documented in the last 6 months before index hospitalization were considered candidates for PIONEER-HF (Table 1). However, it remains speculative that during that 6 -month period and especially at admission, EF was still below $40 \%$. We consider that a diagnosis of HFrEF should be established just before sac/val initiation. For this reason, a heart ultrasound during hospitalization and preferably shortly before sac/val introduction is advisable.

\section{Natriuretic peptides}

Natriuretic peptides have been enthusiastically integrated in everyday clinical practice for risk stratification and guidance of HF management [53]. Recent data from the TRANSITION study show that a favorable NT-proBNP response 4 weeks after sac/val in-hospital initiation is associated with a lower risk for first HF rehospitalization or cardiovascular death [31]. Nevertheless, hospitalized patients with AHF are already at high risk for events, and thus, there is no need to check natriuretic peptides before sac/val initiation [2]. Intriguingly, in a subgroup analysis of PIONEER-HF, it was clearly demonstrated that the clinical efficacy of sac/ val was evident irrespective of baseline natriuretic peptide levels [14]. Taking also into account the non-negligible cost and lack of availability of natriuretic peptide assays in several community and district hospitals, the criterion of PIONEER-HF for elevated natriuretic peptide values before $\mathrm{sac} / \mathrm{val}$ initiation was not included in our modified criteria (Table 1). Implementing natriuretic peptides before or after in-hospital sac/val initiation is optional and it should remain at the discretion of each physician.

\section{Starting dose and up-titration of Sac/Val}

In PIONEER-HF, the starting dose of sac/val was selected on the basis of systolic blood pressure measurements according to a pre-specified dosing algorithm. If the systolic blood pressure was $\geq 120 \mathrm{mmHg}$, an intermediate sac/val dose (49/51 mg) was given, whereas for lower systolic blood pressures $(100-120 \mathrm{mmHg}$ ), patients were started with the lower drug dose (24/26 mg). After discharge, a dose up-titration scheme was followed every week for the first 2 weeks and every second week thereafter with a view to achieving the maximum tolerated dose. From a safety point of view, this strategy resulted in low rates of adverse events with sac/val. However, in a subgroup analysis of PIONEER-HF, sac/val was associated with a higher drop in systolic blood pressure the first week after randomization and a trend towards higher incidence of symptomatic hypotension at week 8 among elderly patients (age $\geq 65$ years) [14].

To minimize the risk of first-dose hypotension, especially in the elderly and in patients with borderline blood pressure and to prevent other serious side effects (i.e., hyperkalemia, renal function worsening) related to sac/val, a revised and more convenient algorithm is shown in Fig. 1.

According to the proposed algorithm, for all hospitalized patients with a systolic blood pressure $\geq 100 \mathrm{mmHg}$, the first dose of sac/val should not exceed 24/26 mg ('low dose approach'). It is advisable to measure the arterial blood pressure $2 \mathrm{~h}$ after the first dose when the maximum effect of the drug is anticipated, and $12 \mathrm{~h}$ after, just before the second dose. Only when systolic blood pressure is higher or equal to $90 \mathrm{mmHg}$ can the second dose be given; otherwise, the drug should be temporarily withheld. Notably, the same cutoff value for systolic blood pressure was selected as a criterion by the investigators of LIFE trial to administer sac/val 
in patients with advanced chronic $\mathrm{HF}^{28}$ After discharge, a $50-100 \%$ increase in the dose is advisable every 1 or 2 weeks until the maximum tolerated dose is reached. Kidney function and potassium levels should always be monitored 1 week after every sac/val dose increase. Close monitoring and both drug and diet modification are usually required when $\mathrm{a} \geq 30 \%$ decrease of eGFR is observed. Based on FDA and EMA labeling, sac/val should be used with caution in patients with severe renal dysfunction (eGFR $15-30 \mathrm{~mL} / \mathrm{min} / 1.73 \mathrm{~m}^{2}$ ) because of a twofold higher exposure to its active metabolite LBQ657. Finally, we recommend careful evaluation and consideration for discontinuation of the drug in cases with significant hyperkalemia (serum potassium $>5.5 \mathrm{mEq} / \mathrm{L}$ ).

\section{Specific concerns and considerations}

\section{Patients on ACE-I before switching to Sac/Val}

For patients receiving ACE-I, a 36-h washout period is mandatory before the initiation of sac/val to reduce the risk of angioedema. Interestingly, both TRANSITION and PIONEER-HF did not report a higher risk for adverse events during washout period. On the contrary, switching to sac/val reduces rapidly NT-proBNP [31] and significantly improves short-term outcomes. [14] In the case of a clinically significant rise in arterial blood pressure during the washout period, low doses of vasodilators and/or calcium channel blockers are recommended. An alternative approach for all sac/val candidates is the direct switch from ACE-I to ARB early in hospitalization. This strategy facilitates sac/val introduction since changing ARB to sac/ val does not require additional waiting times for ARB washout and does not unduly increase length of stay. [5]

\section{Renin-angiotensin-aldosterone system inhibition in naive patients}

Half of the patients recruited in PIONEER-HF and 24\% included in TRANSITION were not exposed to either ACE-I or ARBs prior to hospital admission [14, 29]. Both the safety profile and clinical efficacy of sac/val were comparable among the ACE-I/ARBs naive and non-naive patients [14]. It is worth noting that sac/val resulted in a significantly greater reduction of NT-proBNP in naive patients, which was evidenced within the first week of treatment [36].

These data highlight the significant benefit anticipated from sac/val use in the subset of ACE-I/ARB naive patients and they also mitigate safety concerns that may arise during hospitalization for this subgroup.

\section{B-blockers}

The discontinuation of b-blockers during hospitalization is associated with a higher risk-adjusted mortality [15]. Moreover, short-term mortality rates are lower in AHF patients receiving b-blockers at hospital discharge [15]. For this reason, inhospital initiation and up-titration of b-blockers are mandatory. Evidence-based b-blockers (bisoprolol, metoprolol, carvedilol) are rather more familiar to use in patients with AHF. Theoretically, carvedilol may exert a more potent antihypertensive effect as compared with the selective b1 receptor blockers, because of its alpha adrenergic receptor blocker properties [54] and caution is warranted with concomitant sac/val use. Clinical experience suggests that co-administration of small doses of b-blockers and sac/val in hemodynamically stable and euvolemic patients is safe. In addition, co-administration may also confer the potential advantage of a more effective neurohormonal axis blockade, which translates into a remarkably higher clinical efficacy at least on a theoretical basis. However, a validated algorithm for evidence-based modifying HF medical therapy in AHF with dosing recommendations, priority order, co-administration, and up-titration is still lacking [5].

\section{MRAs}

In PIONEER-HF, only a minority $(\sim 10 \%)$ of patients were on MRAs before randomization and the frequency of prescription of MRAs post-discharge is still unknown. Consequently, conclusions regarding the safety and efficacy of sac/ val and MRA combination during hospitalization for AHF remain speculative. Theoretically, an MRA may confer an additional benefit in patients hospitalized for AHF. Thus, MRAs should be considered for all patients receiving sac/val [15]. However, regular monitoring of potassium and kidney function is compulsory in that case.

\section{Diuretics}

After sac/val administration, the dose of diuretics may need to be readjusted especially for hypovolemic patients. As mentioned above, sac/val potentiates diuresis and this may result in a significant reduction of the effective intravascular volume. This additive diuretic effect can be beneficial for patients with hypervolemia since it may alleviate residual and sometimes latent congestion. However, for hypovolemic patients and especially for those who experience hypotension after sac/val, a reduction of more than $50 \%$ or even withdrawal of the diuretic in a stepwise approach may be required before considering sac/val discontinuation (Fig. 2). After discharge and at follow-up visits, the diuretic dose should be reconsidered on the basis of clinical and laboratory parameters [55].

\section{Management of adverse events during hospitalization}

Arterial hypotension, worsening renal function, and hyperkalemia are the most common adverse events associated with 


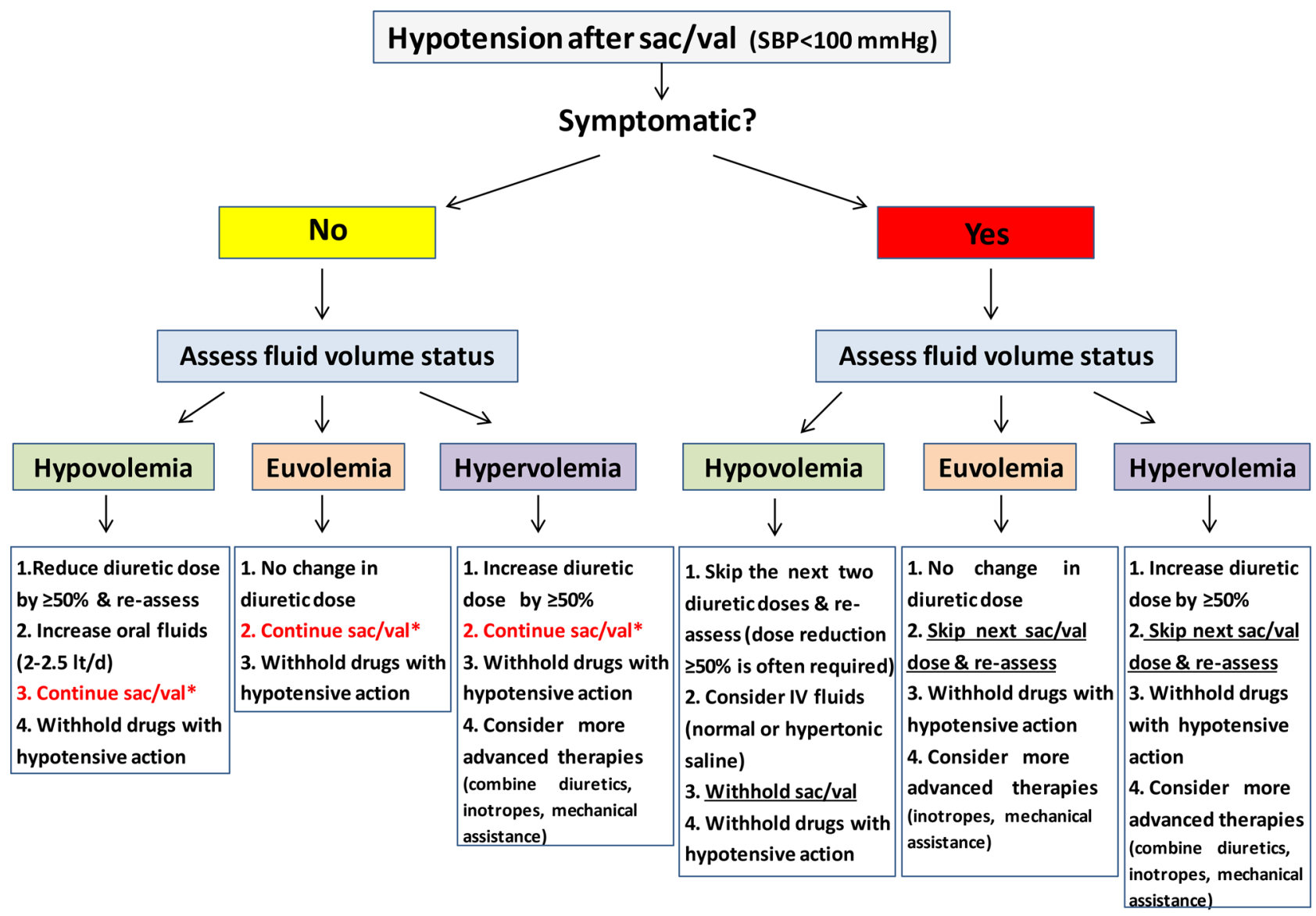

*Only if SBP $<90 \mathrm{mmHg}$, consider temporary discontinuation of sac/val

Fig. 2 Proposed algorithm for sac/val-induced hypotension during hospitalization for AHF. AHF: acute heart failure, BP: blood pressure, SBP: systolic blood pressure, Sac/val: sacubitril/valsartan

sac/val. Surveillance and prompt diagnosis can prevent even more serious complications. It should be noted that a greater reduction of cardiovascular events was observed in patients with chronic HF and low systolic blood pressure treated with sac/val at the expense of a higher incidence of hypotension [56].

In case of hypovolemia and symptomatic hypotension after sac/val, the following general measures should be taken: (a) discontinuation of medications with hypotensive action (i.e., calcium channel blockers, nitrates, etc.), (b) at least $50 \%$ reduction of the dose of diuretics, and (c) administration of oral or intravenous fluid. The next step is to downtitrate (usually by $50 \%$ ) or to withhold sac/val. Conversely, sac/val-induced hypotension in patients with hypervolemia often implies borderline advanced heart failure which may require sac/val discontinuation, inotropic support, and/or mechanical assistance. An algorithm for the management of sac/val-induced hypotension during hospitalization is shown in Fig. 2.
For worsening renal function, withdrawal of nephrotoxic agents and correction of hypovolemia if any should precede any sac/val dose reduction or discontinuation.

Finally, in case of hyperkalemia, suspension of potassium supplements and MRAs and administration of a potassium binder should first be implemented.

\section{SAC/VAL initiation early after discharge}

For patients not considered eligible for sac/val during hospitalization and for those successfully managed in the emergency room or short stay unit, early post-discharge assessment and sac/val initiation in an outpatient care service may confer some benefits. Safety outcome data from the TRANSITION study showed similar rates of side effects between early post-discharge and pre-discharge initiation of sac/val. In addition, efficacy outcome data 
showed that early post-discharge sac/val initiation was not inferior to pre-discharge in terms of attaining the maximum sac/val dose within a 10 -week follow-up time period. [29] These data, together with the positive findings of the open-label extension of PIONEER-HF which showed a dramatic NT-proBNP reduction after switch from enalapril to sac/val 8 weeks post-discharge [36], support the use of sac/val early post-discharge in an outpatient setting.

It is worth emphasizing that patients with AHF should be instructed to visit their attending physician within 1 week after discharge and the hospital cardiology team within 2 weeks [10]. Frequent follow-up appointments should be especially reserved for patients on suboptimal dose or for those not receiving the recommended guideline-directed medical therapies. Early follow-up after discharge may prevent rehospitalizations during the vulnerable phase and improve prognosis. [57]

\section{Conclusions}

In-hospital sac/val initiation in hemodynamically stable patients with HFrEF admitted with an episode of AHF is safe and well-tolerated and results in a greater reduction of NT-proBNP and a significant risk reduction for HF rehospitalizations. However, the lack of sufficient clinical experience, ambiguities and pitfalls in defining clinically stable patients, and concerns regarding imminent adverse events are significant obstacles for sac/val in-hospital use. Implementation of the comprehensive and easily applicable algorithm proposed for in-hospital sac/val initiation can improve patient access to sac/val therapy during hospitalization. Prospective studies are warranted to explore further the validity, applicability, and clinical impact of our consensus eligibility criteria and algorithm.

\section{Declarations}

Conflict of interest The authors state that there is no conflict of interest regarding this work, although in the past, Dr Christina Chrysohoou, Dr Argyrios Ntalianis, Dr Katerina Naka, and Dr Gregory Giamouzis have received honoraria for lectures from Novartis.

Additionally, John Parissis has received honoraria for lectures from Servier, Pfizer, Novartis, Orion Pharma, and Astra Zeneca

\section{References}

1. Conrad N, Judge A, Tran J, Mohseni H, Hedgecott D, Crespillo AP, Allison M, Hemingway H, Cleland JG, McMurray JJV, Rahimi K (2018) Temporal trends and patterns in heart failure incidence: a population-based study of 4 million individuals. Lancet 391:572-580
2. Seferovic PM, Ponikowski P, Anker SD, Bauersachs J, Chioncel O, Cleland JGF, de Boer RA, Drexel H, Ben Gal T, Hill L, Jaarsma T, Jankowska EA, Anker MS, Lainscak M, Lewis BS, McDonagh T, Metra M, Milicic D, Mullens W, Piepoli MF, Rosano G, Ruschitzka F, Volterrani M, Voors AA, Filippatos G, Coats AJS (2019) Clinical practice update on heart failure 2019: pharmacotherapy, procedures, devices and patient management. An expert consensus meeting report of The Heart Failure Association of the European Society of Cardiology. Eur J Heart Fail 21:1169-1186

3. Filippatos G, Farmakis D, Bistola V, Karavidas A, Mebazaa A, Maggioni AP, Parissis J (2014) Temporal trends in epidemiology, clinical presentation and management of acute heart failure: results from the Greek cohorts of the Acute Heart Failure Global Registry of Standard Treatment and the European Society of Cardiology Heart Failure pilot survey. Eur Heart J Acute Cardiovasc Care. https://doi.org/10.1177/2048872614527012

4. Maggioni AP, Dahlström U, Filippatos G, Chioncel O, Crespo Leiro M, Drozdz J, Fruhwald F, Gullestad L, Logeart D, Fabbri G, Urso R, Metra M, Parissis J, Persson H, Ponikowski P, Rauchhaus M, Voors AA, Nielsen OW, Zannad F, Tavazzi L (2013) EURObservational Research Programme: regional differences and 1-year follow-up results of the Heart Failure Pilot Survey (ESC-HF Pilot). Heart Failure Association of the European Society of Cardiology (HFA). Eur J Heart Fail 15:808-817

5. Hollenberg SM, Stevenson LW, Ahmad T, Amin VJ, Bozkurt B, Butler J, Davies L, Drazner MH, Kirkpatrick JN, Peterson PN, Reed BN, Roy CL, Storrow AB (2019) 2019 ACC Expert Consensus Decision Pathway on risk assessment, management, and clinical trajectory of patients hospitalized with heart failure. J Am Coll Cardiol 74:1966-2011

6. Sanam K, Bhatia V, Bajaj NS, Gaba S, Morgan CJ, Fonarow GC, Butler J, Deedwania P, Prabhu SD, Wu WC, White M, Love TE, Aronow WS, Fletcher RD, Allman RM, Ahmed A (2016) Reninangiotensin system inhibition and lower 30-day all-cause readmission in Medicare Beneficiaries with heart failure. Am J Med 129(10):1067-1073

7. Fonarow GC, Abraham WT, Albert NM, Stough WG, Gheorghiade M, Greenberg BH, O'Connor CM, Sun JL, Yancy C, Young JB (2007) Carvedilol use at discharge in patients hospitalized for heart failure is associated with improved survival: an analysis from Organized Program to Initiate Lifesaving Treatment in Hospitalized Patients with Heart Failure (OPTIMIZE-HF). Am Heart J 153:82e1-e11

8. Jondeau G, Neuder Y, Eicher JC, Jourdain P, Fauveau E, Galinier M, Jegou A, Bauer F, Trochu JN, Bouzamondo A, Tanguy ML, Lechat P (2009) B-CONVINCED: beta-blocker CONtinuation Vs. interruption in patients with Congestive heart failure hospitalizED for a decompensation episode. Eur Heart J 30:2186-2192

9. Bistola V, Simitsis P, Farmakis D, Ikonomidis I, Bakosis G, Triposkiadis F, Hatziagelaki E, Lekakis J, Mebazaa A, Parissis J (2018) Association of mineralocorticoid receptor antagonist use and in-hospital outcomes in patients with acute heart failure. Clin Res Cardiol 107:76-86

10. Ponikowski P, Voors AA, Anker SD, Bueno H, Cleland JGF, Coats AJS, Falk V, Gonzalez-Juanatey JR, Harjola VP, Jankowska EA, Jessup M, Linde C, Nihoyannopoulos P, Parissis JT, Pieske B, Riley JP, Rosano GMC, Ruilope LM, Ruschitzka F, Rutten FH, van der Meer P, Group ESCSD (2016) 2016 ESC Guidelines for the diagnosis and treatment of acute and chronic heart failure: The Task Force for the diagnosis and treatment of acute and chronic heart failure of the European Society of Cardiology (ESC)Developed with the special contribution of the Heart Failure Association (HFA) of the ESC. Eur Heart J 37(27):2129-2200

11. Yancy CW, Jessup M, Bozkurt B et al (2017) 2017 ACC/AHA/ HFSA focused update of the 2013 ACCF/AHA guideline for the management of heart failure: a report of the American College of Cardiology/American Heart Association Task Force on Clinical 
Practice Guidelines and the Heart Failure Society of America. Circulation 136(6):e137-e161

12. Kozhuharov N, Goudev A, Flores D, Maeder MT, Walter J, Shrestha S, Gualandro DM, de Oliveira MT Jr, Sabti Z, Müller B, Noveanu M, Socrates T, Ziller R, Bayés-Genís A, Sionis A, Simon P, Michou E, Gujer S, Gori T, Wenzel P, Pfister O, Conen D, Kapos I, Kobza R, Rickli H, Breidthardt T, Münzel T, Erne P, Mueller C, GALACTIC Investigators, Mueller C, Erne P, Müller B, Rickli H, Maeder M, de Oliveira MT Jr, Bayés-Genís A, Sionis A, Goudev A, Dimov B, Hartwiger S, Arenja N, Glatz B, Herr N, Isenrich R, Mosimann T, Twerenbold R, Boeddinghaus J, Nestelberger T, Puelacher C, Freese M, Vögele J, Meissner K, Martin J, Strebel I, Wussler D, Schumacher C, Osswald S, Vogt F, Hilti J, Barata S, Schneider D, Schwarz J, Fitze B, Hartwiger S, Arenja N, Glatz B, Herr N, Isenrich R, Mosimann T, Rentsch K, Bossa A, Jallad S, Soeiro A, Georgiev D, Jansen T, Gebel G, Bossard M, Christ M (2019) Effect of a Strategy of Comprehensive Vasodilation vs Usual Care on Mortality and Heart Failure Rehospitalization Among Patients With Acute Heart Failure: The GALACTIC Randomized Clinical Trial. JAMA 322(23):2292-2302

13. Rosenblum H, Kapur N, Abraham W, Udelson J, Itkin M, Uriel N, Voors A, Burkhoff D (2020) Conceptual Considerations for Device-Based Therapy in Acute Decompensated Heart Failure: DRI 2 P 2 S. Circ Heart Fail 13(4):e006731. https://doi.org/10. 1161/CIRCHEARTFAILURE.119.006731 (Epub 2020 Apr 16)

14. Velazquez EJ, Morrow DA, DeVore AD, Duffy C, Ambrosy A, McCague K, Rocha R, Braunwald E, Investigators PIONEER-HF (2019) Angiotensin-neprilysin inhibition in acute decompensated heart failure. N Engl J Med 380(6):539-548

15. Bhagat A, Greene S, Vaduganathan M, Fonarow G, Butler J (2019) Initiation, Continuation, Switching, and Withdrawal of Heart Failure Medical Therapies During Hospitalization. JACC Heart Fail 7(1):1-12. https://doi.org/10.1016/j.jchf.2018.06.011

16. Allen L, Fonarow G, Liang L, Schulte P, Masoudi F, Rumsfeld J, Ho M, Eapen Z, Hernandez A, Heidenreich P, Bhatt D, Peterson E, Krumholz H, on behalf of the American Heart Association's Get With The Guidelines Heart Failure (GWTG-HF) Investigators (2015) Medication Initiation Burden Required to Comply with Heart Failure Guideline Recommendations and Hospital Quality Measures. Circulation 132(14):1347-1353

17. Farmakis D, Parissis J, Karavidas A, Karvounis C, Triposkiadis F, Filippatos G, Lekakis J (2015) In-hospital management of acute heart failure: Practical recommendations and future perspectives. Int J Cardiol 201:231-236

18. Arvanitaki A, Michou E, Kalogeropoulos A, Karvounis H, Giannakoulas G (2020) Mildly symptomatic heart failure with reduced ejection fraction: diagnostic and therapeutic considerations. ESC Heart Fail 7(4):1477-1487

19. McMurray JJ, Packer M, Desai AS, Gong J, Lefkowitz MP, Rizkala AR, Rouleau JL, Shi VC, Solomon SD, Swedberg K, Zile MR, Investigators Committees PH (2014) Angiotensin-neprilysin inhibition versus enalapril in heart failure. N Engl J Med 371(11):993-1004

20. Desai AS, Solomon SD, Shah AM, Claggett B, Fang J, Izzo J, McCague K, Abbas C, Rocha R, Mitchell G (2019) Effect of SacubitrilValsartan vs Enalapril on Aortic Stiffness in Patients With Heart Failure and Reduced Ejection Fraction: A Randomized Clinical Trial [published online ahead of print, 2019 Sep 2]. JAMA 322(11):1-10

21. D'Elia E, Iacovoni A, Vaduganathan M, Lorini FL, Perlini S, Senni M (2017) Neprilysin inhibition in heart failure: mechanisms and substrates beyond modulating natriuretic peptides. Eur J Heart Failure 19:710-717

22. Zile MR, O'Meara E, Claggett B, Prescott M, Solomon S, Swedberg K, Packer M, McMurray J, Shi V, Lefkowitz M, Rouleay J (2019) Effects of sacubitril/valsartan on biomarkers of extracellular matrix regulation in patients with HFrEF. J Am Coll Cardiol 73(7):795-806
23. Januzzi JL Jr, Prescott MF, Butler J, Felker GM, Maisel AS, McCague K, Camacho A, Piña IL, Rocha RA, Shah AM, Williamson KM, Solomon SD, Investigators PROVE-HF (2019) Association of Change in N-Terminal Pro-B-Type Natriuretic Peptide Following Initiation of Sacubitril-Valsartan Treatment With Cardiac Structure and Function in Patients With Heart Failure With Reduced Ejection Fraction. JAMA 322(11):1-11. https://doi.org/ 10.1001/jama.2019.12821

24. Damman K, Gori M, Claggett B, Jhund PS, Senni M, Lefkowitz MP, Prescott MF, Shi VC, Rouleau JL, Swedberg K, Zile MR, Packer M, Desai AS, Solomon SD, McMurray JJV (2018) Renal effects and associated outcomes during angiotensin-neprilysin inhibition in heart failure. JACC Heart Fail 6(6):489-498

25. Seferovic JP, Claggett B, Seidelmann SB, Seely EW, Packer M, Zile MR, Rouleau JL, Swedberg K, Lefkowitz M, Shi VC, Desai AS, McMurray JJV, Solomon SD (2017) Effect of sacubitril/valsartan versus enalapril on glycaemic control in patients with heart failure and diabetes: a post-hoc analysis from the PARADIGM-HF trial. Lancet Diabetes Endocrinol 5(5):333-340

26. Desai AS, Vardeny O, Claggett B, McMurray JJ, Packer M, Swedberg K, Rouleau JL, Zile MR, Lefkowitz M, Shi V, Solomon SD (2017) Reduced risk of hyperkalemia during treatment of heart failure with mineralocorticoid receptor antagonists by use of sacubitril/valsartan compared with enalapril: a secondary analysis of the PARADIGMHF Trial. JAMA Cardiol 2(1):79-85

27. Maddox TM, Januzzi JL Jr, Allen LA, Breathett K, Butler J, Davis LL, Fonarow GC, Ibrahim NE, Lindenfeld J, Masoudi FA, Motiwala SR, Oliveros E, Patterson JH, Walsh MN, Wasserman A, Yancy CW, Youmans QR (2021) 2021 Update to the 2017 ACC Expert Consensus Decision Pathway for Optimization of Heart Failure Treatment: Answers to 10 Pivotal Issues About Heart Failure With Reduced Ejection Fraction: A Report of the American College of Cardiology Solution Set Oversight Committee. J Am Coll Cardiol 77(6):772-810

28. Mann DL, Greene SJ, Givertz MM, Vader JM, Starling RC, Ambrosy AP, Shah P, McNulty SE, Mahr C, Gupta D, Redfield MM, Lala A, Lewis GD, Mohammed SF, Gilotra NA, DeVore A, Gorodeski EZ, Desvigne-Nickens P, Hernandez AF, Braunwald E (2020) Sacubitril/Valsartan in Advanced Heart Failure With Reduced Ejection Fraction: Rationale and Design of the LIFE Trial. JACC Heart Fail 8(10):789-799

29. Wachter R, Senni M, Belohlavek J, Straburzynska-Migaj E, Witte KK, Kobalava Z, Fonseca C, Goncalvesova E, Cavusoglu Y, Fernandez A, Chaaban S, Bøhmer E, Pouleur AC, Mueller C, Tribouilloy C, Lonn E, A L Buraiki J, Gniot J, Mozheiko M, Lelonek M, Noè A, Schwende H, Bao W, Butylin D, Pascual-Figal D; TRANSITION Investigators. (2019) Initiation of sacubitril/ valsartan in haemodynamically stabilised heart failure patients in hospital or early after discharge: primary results of the randomised TRANSITION study. Eur J Heart Fail 21(8):998-1007

30. Senni M, Wachter R, Witte K, Straburzynska-Migaj E, Belohlavek J, C, Mueller C, Lonn E, Chakrabarti A, Bao W, Noe A, Schwende $\mathrm{H}$, Butylin D, Pascual-Figal D, TRANSITION Investigators. (2020) Initiation of sacubitril/valsartan shortly after hospitalisation for acutely decompensated heart failure in patients with newly diagnosed (de novo) heart failure: a subgroup analysis of the TRANSITION study. Eur J Heart Fail 22(2):303-312

31. Pascual-Figal D, Wachter R, Senni M, Bao W, Noe A, Schwende H, Butylin D, Prescott M (2020) NT-proBNP Response to Sacubitril/Valsartan in Hospitalized Heart Failure Patients With Reduced Ejection Fraction: TRANSITION Study. JACC Heart Fail S2213-1779(20)30336-X

32. Ambrosy AP, DeVore AD, Velazquez EJ (2019) In-hospital initiation of sacubitril/valsartan in acute decompensated heart failure: being in the right place at the right time. Eur J Heart Fail 21(8):1008-1011 
33. Morrow DA, Velazquez EJ, DeVore AD, Prescott M, Duffy C, Gurmu Y, McCague K, Rocha R, Braunwald E (2019) Cardiovascular biomarkers in patients with acute decompensated heart failure randomized to sacubitril-valsartan or enalapril in the PIONEER-HF trial. Eur Heart J 40(40):3345-3352

34. Morrow DA, Velazquez EJ, DeVore AD, Desai AS, Duffy CI, Ambrosy AP, Gurmu Y, McCague K, Rocha R, Braunwald E (2019) Clinical Outcomes in Patients With Acute Decompensated Heart Failure Randomly Assigned to Sacubitril/Valsartan or Enalapril in the PIONEER-HF Trial. Circulation 139(19):2285-2288

35. Berg DD, Braunwald E, DeVore AD, Lala A, Pinney SP, Duffy CI, Gurmu Y, Velazquez EJ, Morrow DA (2020) Efficacy and Safety of Sacubitril/Valsartan by Dose Level Achieved in the PIONEERHF Trial. JACC Heart Fail S2213-1779(20)30384-X

36. Ambrosy AP, Braunwald E, Morrow DA, De Vore A, McCague K, Meng X, Duffy C, Rocha R, Velazquez E (2020) Angiotensin receptor-neprilysin inhibition based on history of heart failure and use of renin-angiotensin system antagonists. J Am Coll Cardiol 76(9):1034-1048

37. DeVore AD, Braunwald E, Morrow DA, Duffy C, Ambrosy A, Chakraborty H, McCague K, Rocha R, Velazquez E (2019) Initiation of Angiotensin-Neprilysin Inhibition After Acute Decompensated Heart Failure: Secondary Analysis of the Open-label Extension of the PIONEER-HF Trial [published online ahead of print, Dec 11]. JAMA Cardiol 5(2):202-207

38. Gaziano TA, Fonarow GC, Velazquez EJ, Morrow DA, Braunwald E, Solomon SD (2020) Cost-effectiveness of Sacubitril-Valsartan in Hospitalized Patients Who Have Heart Failure With Reduced Ejection Fraction. JAMA Cardiol 5(11):1236-1244

39. Greene SJ, Triana TS, Ionescu-Ittu R, Burne R, Guerin A, Borentain M, Kessler P, Tugsu A, DeSouza M, Felker M, Chen L. (2020) In-Hospital Therapy for Heart Failure With Reduced Ejection Fraction in the United States [published online ahead of print, Aug 12]. JACC Heart Fail S2213-1779(20)30383-8

40. Srivastava P, Fonarow G (2019) In-Hospital Initiation of Angiotensin Receptor-Neprilysin Inhibitors-The Time Is Now. JAMA Cardiol 4(3):195-196. https://doi.org/10.1001/jamacardio.2019.0104

41. Fudim M, Sayeed S, Xu H, Matsouaka RA, Heidenreich PA, Velazquez EJ, Yancy CW, Fonarow GC, Hernandez AF, DeVore AD (2020) Representativeness of the PIONEER-HF Clinical Trial Population in Patients Hospitalized With Heart Failure and Reduced Ejection Fraction. Circ Heart Fail 13(4):e006645

42. Chioncel O, Mebazaa A, Maggioni AP, Harjola VP, Rosano G, Laroche C, Piepoli M, Crespo-Leiro M, Lainscak M, Ponikowski P, Fillipatos G, Ruschitzka F, Seferovic P, Coats A, Lund L (2019) Acute heart failure congestion and perfusion status - impact of the clinical classification on in-hospital and long-term outcomes; insights from the ESC-EORP-HFA Heart Failure Long-Term Registry. Eur J Heart Fail 21(11):1338-1352. https://doi.org/10.1002/ejhf.1492

43. Antoniou CK, Chrysohoou C, Lerakis S, Manolakou P, Pitsavos C, Tsioufis K, Stefanadis C, Tousoulis D (2015) Effects of ventriculoarterial coupling changes on renal function, echocardiographic indices and energy efficiency in patients with acute decompensated systolic heart failure under furosemide and dopamine treatment: a comparison of three therapeutic protocols. Int J Cardiol 199:44-49

44. Triposkiadis FK, Butler J, Karayannis G, Starling R, Fillipatos G, Wolski K, Parissis J, Parisis C, Rovithis D, Koutrakis K, Skoularigis J, Antoniou CK, Chrysohoou C, Pitsavos C, Stefanadis C, Nastas J, Tsaknakis T, Mantziari L, Giannakoulas G, Karvounis H, Kalogeropoulos A, Giamouzis G (2014) Efficacy and safety of high dose versus low dose furosemide with or without dopamine infusion: the Dopamine in Acute Decompensated Heart Failure II (DAD-HF II) trial. Int J Cardiol 172(1):115-121
45. Javaloyes P, Miró Ò, Gil V, Martin-Sanchez FJ, Jacob J, Herrero P, Takagi K, Alquezar-Arbe A, Lopez Diez MP, Martin E, Bibiano C, Escoda R, Gil C, Fuentes M, Llopis Garcia G, Alvarez Perez JM, Jerez A, Tost J, Llauger L, Romero R, Garido JM, RodriguezAdrada E, Sanchez C, Rossello X, Parissis J, Mebazza A, Chioncel O, Llorens P (2019) Clinical phenotypes of acute heart failure based on signs and symptoms of perfusion and congestion at emergency department presentation and their relationship with patient management and outcomes. Eur J Heart Fail 21(11):1353-1365

46. Ochiai ME, Brancalhão EC, Puig RS, Vieira KR, Cardoso JN, Oliveira MT Jr, Barretto AC (2014) Short-term add-on therapy with angiotensin receptor blocker for end-stage inotrope-dependent heart failure patients: B-type natriuretic peptide reduction in a randomized clinical trial. Clinics (Sao Paulo) 69(5):308-313

47. Mullens W, Damman K, Harjola VP, Mebazaa A, Brunner-La Rocca HP, Martens P, Jeffrey Testani J, Tang W, Orso F, Rossignol P, Metra M, Filippatos G, Seferovic P, Ruschitzka F, Coats A (2019) The use of diuretics in heart failure with congestion - a position statement from the Heart Failure Association of the European Society of Cardiology. Eur J Heart Fail 21(2):137-155. https://doi.org/ 10.1002/ejhf.1369 (Epub 2019 Jan 1)

48. Lancellotti P, Galderisi M, Edvardsen T, Donal E, Goliasch G, Cardim N, Magne J, Laginha S, Hagendorff A, Haland TF, Aaberge L, Martinez C, Rapacciuolo A, Santoro C, Ilardi F, Postolache A, Dulgheru R, Mateescu AD, Beladan CC, Deleanu D, Marchetta S, Auffret V, Schwammenthal E, Habib G, Popescu BA (2017) Echo Doppler estimation of left ventricular filling pressure: results of the multicentre EACVI Euro-Filling study. Eur Heart J Cardiovasc Imaging 18(9):961-968

49. McCallum W, Tighiouart H, Testani J, Griffin M, Konstam M, Udelson J, Sarnak M (2020) Acute kidney function declines in the context of decongestion in acute decompensated heart failure. JACC Heart Fail 8(7):537-547

50. Guarracino F, Zima E, Pollesello P, Masip J (2020) Short term treatments for acute cardiac care: inotropes and inodilators. Eur Heart J Suppl 22(Suppl D):D3-D11

51. Felker GM, Ellison DH, Mullens W, Cox ZL, Testani JM (2020) Diuretic Therapy for Patients With Heart Failure: JACC State-ofthe-Art Review. J Am Coll Cardiol 75(10):1178-1195

52. Stevenson LW (2003) Clinical Use of Inotropic Therapy for Heart Failure: Looking Backward or Forward? Part I: Inotropic Infusions During Hospitalization. Circulation 108(3):367-372

53. Maisel A, Mueller C, Adams K Jr, Anker SD, Aspromonte N, Cleland JG, Cohen-Solal A, Dahlstrom U, DeMaria A, Di Somma S, Filippatos GS, Fonarow GC, Jourdain P, Komajda M, Liu PP, McDonagh T, McDonald K, Mebazaa A, Nieminen MS, Peacock WF, Tubaro M, Valle R, Vanderhyden M, Yancy CW, Zannad F, Braunwald E (2008) State of the art: using natriuretic peptide levels in clinical practice. Eur J Heart Fail 10(9):824-839

54. Kotecha D, Holmes J, Krum H, Altman DG, Manzano L, Cleland JG, Lip GY, Coats AJ, Andersson B, Kirchhof P, von Lueder TG, Wedel H, Rosano G, Shibata MC, Rigby A, Flather MD (2014) Beta-Blockers in Heart Failure Collaborative G. Efficacy of beta blockers in patients with heart failure plus atrial fibrillation: an individual-patient data meta-analysis. Lancet 384(9961):2235-2243

55. Eid PS, Ibrahim DA, Zayan AH, Abdelrahman MM, Shehata MAA, Kandil H, Abouibrahim MA, Duy LM, Shinkar A, Elfaituri MK, Minh LHN, Fahmy MM, Tam DNH, Vuong NL, Shah J, Do VBD, Hirayama K, Huy NT (2020) Comparative effects of furosemide and other diuretics in the treatment of heart failure: a systematic review and combined meta-analysis of randomized controlled trials [published online ahead of print, Aug 11]. Heart Fail Rev https:// doi.org/10.1007/s10741-020-10003-7 
56. Böhm M, Young R, Jhund PS, Solomon S, Gong J, Lefkowitz M, Rizkala A, Rouleau J, Shi V, Swedberg K, Zile M, Packer M, McMurray J (2017) Systolic blood pressure, cardiovascular outcomes and efficacy and safety of sacubitril/valsartan (LCZ696) in patients with chronic heart failure and reduced ejection fraction: results from PARADIGM-HF. Eur Heart J 38(15):1132-1143. https://doi.org/10.1093/eurheartj/ehw570
57. McAlister F, Youngson E, Kaul P, Ezekowitz J (2016) Early FollowUp After a Heart Failure Exacerbation. The Importance of Continuity. Circ Heart Fail 9(9):e003194. https://doi.org/10.1161/CIRCH EARTFAILURE.116.003194

Publisher's Note Springer Nature remains neutral with regard to jurisdictional claims in published maps and institutional affiliations. 